\title{
Time-delay measurements depending on lasing wavelengths of single small-size droplet dye laser
}

\author{
Hiroshi Taniguchi \\ Department of Electrical and Electronic Engineering, Faculty of Engineering, Iwate University, 4-3-5 Ueda, \\ Morioka 020, Japan \\ Humihiro Tomisawa \\ Department of Electrical Engineering, Faculty of Engineering, Iwate University, 4-3-5 Ueda, Morioka 020, \\ Japan
}

(Received 20 April 1995; accepted for publication 24 August 1995)

\begin{abstract}
Measurements of time delay of several nanoseconds depending on the lasing wavelengths from $200-\mu \mathrm{m}$-diam single-droplet dye laser are reported. The wavelength tuning is performed by injecting scattering particles into the droplet dye laser. It is suggested that the time delay depending on the lasing wavelengths by varying the quantity of the scatterers is related to the round-trip time required to support lasing in the droplet. (C) 1995 American Institute of Physics.
\end{abstract}

Recently, many investigations have interested several workers on the light-scattering properties of dielectric spheres. The advantages of the spherical resonator have been pointed out as the very high quality factor ( $Q$ value) of electromagnetic whispering-gallery mode $(W G M)^{1}$ or morphology-dependent resonances (MDRs) in the dielectric sphere with micrometer-sized droplets ${ }^{2,3}$ or solid particles. ${ }^{4,5}$ In addition, the high- $Q$ value of spherical cavities have been shown to provide very low-threshold lasers. ${ }^{2,3}$ Several recent papers have reported lasing and/or stimulated Raman scattering (SRS) from dye-doped and/or nondoped microdroplets, using this high- $Q$ value to MDRs. ${ }^{6-11}$

Previous investigations of dye-doped solid microsphere lasers that are due to MDRs (or WGM) ${ }^{5,12-15}$ have demonstrated the usefulness for practical application; however, solid sphere lasers may produce other problems such as degradation of the unique $Q$ value in the sphere-surrounding media interface and/or the losses due to adsorption of dye molecules onto the sphere surface..$^{9-11}$

Spectral measurements of lasing and SRS reveal the existence of quasiperiodic peaks that are identified through MDRs of the irradiated droplets. ${ }^{6,7}$ Time-resolved measurements of lasing and SRS exhibit characteristics not found in the corresponding bulk spectra. These include faster rise times for lasing emission ${ }^{6}$ and delays in the initiation of SRS. ${ }^{6,7,16}$

In contrast to the above, experimental measurements presented here were carried out from the viewpoint that observable time delays are due to differences in the stimulation processes with cavity $Q$ values of the sphere, which can be controlled by varying the quantity of scatterers into the sphere. $^{17}$

In this letter we report experimental measurements of time delays depending on the lasing wavelengths due to the spherical dye laser containing the injected scattering particles.

The experimental arrangement (see also Fig. 1) and the basic concept for wavelength tuning of spherical liquid dye lasers, are similar to previous report. ${ }^{17}$ The experiment employs a frequency-doubled, $Q$-switched $\mathrm{Nd}$ :YAG laser (Nd:YAG) second harmonic $(\mathrm{SH}) ; 532 \mathrm{~nm}$ with $\sim 3 \mathrm{~mJ}$ pulse energy and $\sim 10 \mathrm{~ns}$ pulse duration. Green light from the Nd:YAG SH $(532 \mathrm{~nm})$ is focused onto dye [rhodamine $6 \mathrm{G}$ (RH-6G)]-doped liquid spheres (DD) that scatter elastic (532 $\mathrm{nm}$ ) and inelastic (redshifted dye lasing) light into an optical multichannel analyzer (OMA; not shown). The weakly focused pump-beam size was large compared to the DD sphere. Spectra of light emitted from the DD sphere are collected at $90^{\circ}$ to the incident pump beam (INC; in the inset of Fig. 1) and are analyzed with an optical multichannel analyzer mounted on a microscope [in a vertical plane; V (OMA) in the inset]. Glycerol/ethanol-containing dye aerosols are successively ejected from a modified atomizer to proper-sized droplets around a fine horizontally strung tungsten fiber (FW: in the inset) of $\sim 5-7 \mu \mathrm{m}$ diam. The use of glycerol-ethanol mixture (of a composition ratio of $\sim 5: 1$ by volume) is for droplet-size stability throughout the experiments. $^{17}$

The wavelength tuning is performed by injecting scattering particles into the droplet dye laser. The lower cavity $Q$ value in presence of scattering particles leads to lasing at shorter wavelengths, corresponding to the larger population inversion (gain) to reach the lasing threshold. The results of the wavelength tuning have been explained, ${ }^{17}$ based on a cavity $Q$ value in terms of a general wavelength dependence of dye lasing determined by gain/loss characteristics, in the droplet dye lasers.

Combining with the experimental procedure mentioned above, the measuring system of time delays between the light pulse signal $\left(I_{s}\right)$ emitted from the dye-doped sphere (DD) laser and direct pumping-Nd:YAG SH pulse $\left(I_{p}\right)$ is shown in Fig. 1. Waveforms are measured using a calibrated Hamamatsu Photonics R1193U-02 biplanar phototube (PT) and a Hewlett-Packard 1744A storage oscilloscope (100 $\mathrm{MHz}$ ) (OSC) system. To separate the two pulses $\left(I_{s}\right.$ and $\left.I_{p}\right)$ on the time scale of the OSC, the reference pulse $\left(I_{p}\right.$; which is the direct pumping pulse of the Nd:YAG SH) travels a relatively short geometrical path through an optical fiber (OF-1), before arriving at the photosensitive surface of the PT. The light pulse emitted from the DD $\left(I_{s}\right)$, which is detected at $\sim 120^{\circ}$ to the incident pumping beam (INC; in the inset) [in a horizontal plane; H(OSC) in the inset of Fig. 1], 


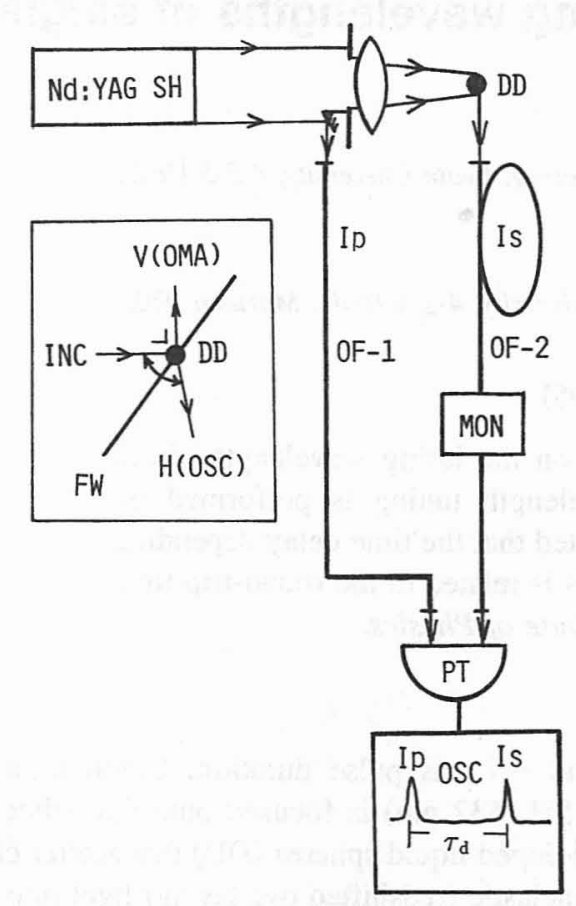

FIG. 1. Experimental arrangement to measure the time difference $\left(\tau_{d}=\tau_{s}-\tau_{P}\right)$ in the droplet dye laser. $I_{p}$ : direct pumping-Nd:YAG SH pulse; $I_{s}$ : light pulse signal emitted from DD; PT: phototube; OSC: oscilloscope; OF-1/OF-2: optical fibers for $I_{p} / I_{s}$ pulse transmission; MON: monochromator

is also transmitted through a monochromator (MON) and an optical fiber (OF-2) of length with suitable delays for separation of the two pulses. Wavelength setting of the MON is established according to the central wavelength displayed on the computer monitor using the OMA. Then we can measure temporal characteristics between $I_{p}$ and $I_{s}$, for given wavelengths of the $I_{s}$ pulses.

The time difference $\left(\tau_{d}\right)$ is defined as $\tau_{d}=\tau_{s}-\tau_{P}$, where $\tau_{P}$ and $\tau_{s}$ are the times at the peak values of $I_{p}$ and $I_{s}$ pulses, respectively. Measurements of $\tau_{d}$ were carried out with normalization to $\tau_{p}$ (corresponding $I_{p}$ is the reference pulse, under these conditions). Smaller (or larger) $\tau_{d}$ corresponds to shorter (or longer) round-trip time in the DD droplet.

Figure 2 shows typical results obtained from the single RH-6G-doped DD $\left(\sim 10^{-3} \mathrm{M}\right.$ concentration and $d=200 \mu \mathrm{m}$ diam), without [Fig. 2(a)]/with [Figs. 2(b)-2(d)] cases of the seeding of artificial scattering spherical particles [poly(methyl methacrylate) (PMMA) with $\sim 10 \mu \mathrm{m}$ diam] into the droplet. Figure 2 comes from the same single droplet by the Nd:YAG SH pumping, without seeding [Fig. 2(a)], with seeding; of $\sim 1 / 16$ PMMA volume of the initial droplet volume [Fig. 2(b)], of $\sim 1 / 8$ volume [Fig. 2(c)], and of $\sim 1 / 4$ volume [Fig. 2(d)], and the central wavelengths of lasing are $605,591,580,568 \mathrm{~nm}$, respectively. The concentration of PMMA was estimated from estimating the total numbers of PMMA (with diameter of $\sim 10 \mu \mathrm{m}$ and specific gravity of 1.19), by measuring the total weights of PMMA. The pumping intensities were held nearly constant with full high amplitude above the lasing threshold, but there was no laserinduced breakdown, because the pumping intensities were

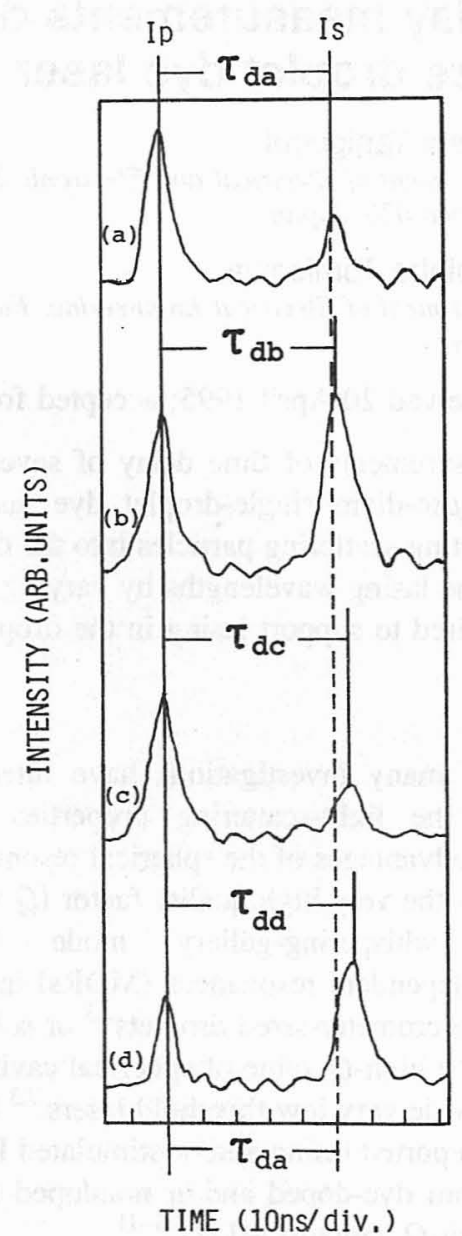

FIG. 2. Comparison of $\tau_{d}$ obtained from single RH-6G-doped sphere $\left(\sim 10^{-3} \mathrm{M}\right.$ concentration, $d=200 \mu \mathrm{m}$ diam), by Nd:YAG SH $(532 \mathrm{~nm})$ pumping, under nearly constant pumping intensities; (a) without PMMA seeding, (b), (c), and (d) ( 1/16 PMMA volume of the initial droplet volume, 1/8, and 1/4) with gradual increasing PMMA seeding in the droplets. $\tau_{d i}$ denotes the $\tau_{d}$ value, where $i$ implies a, b, c, or d corresponding to (a), (b), (c), or (d), respectively. This notation is also used in Fig. 3.

lowered by the use of neutral-density filters. All the figures in Fig. 2 are at the same positions of $\tau_{P}$, for the sake of comparison. It is found from these figures that the value of $\tau_{d}$ increases as the injected scatterers into the initial droplet increases (from the top to the bottom).

The relationship between the lasing wavelength and the time difference exhibited in Fig. 2 (including other experimental data plotted as open circles) is shown in Fig. 3, where the solid circles with (a)-(d) correspond to (a)-(d) in Fig. 2, respectively. In Fig. 3, the vertical axis is scaled in the time difference $\left(\tau_{d i}-\tau_{d a}\right.$; where $i$ implies the cases of (a)-(d) in Fig. 2 or in other experimental data) relative to the smallest $\tau_{d}$ value in our experiments (i.e., with a reference point of $\tau_{d a}$ ). These findings suggest that shorter (or longer) roundtrip times are required to support lasing at longer (or shorter) wavelengths.

We intend to give a simple physical meaning of the present experimental results according to Ref. 17 (with the blueshifts in the dye lasing spectra resulting from the existence of scattering particles), by use of the gain-frequency (inverse-wavelength) characteristics calculated by Weber and 


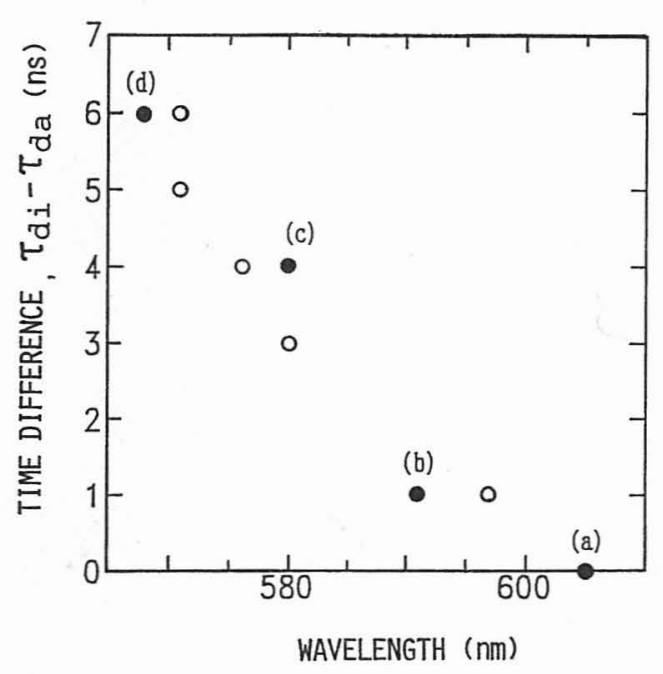

FIG. 3. Lasing-wavelength vs time-difference characteristics obtained in the experiments. Plots with (a)-(d) (solid circles) correspond to (a) -(d) in Fig. 2 , respectively.

Bass. ${ }^{18}$ The result is as follows: (1) The gain-frequency characteristics denote that the position of peak gain with increase of the excited-population shifts toward higher frequency (the shorter wavelength). (2) Laser emission arises at (angular) frequency $\omega$ when the laser gain $G(\omega)$ equal the cavity loss $L(\omega)$, which is determined by losses that result from reflection, diffraction, scattering, and absorption of the laser beam in the optical cavity. We deduce that low (or high) gain is necessary for lasing to satisfy the relation $G(\omega)=L(\omega)$ when the cavity has a high (or low) $Q$ value, where the corresponding frequency is low (or high). Consequently, dye lasing occurs at longer (or shorter) wavelengths when the cavity $Q$ value is high (or low). In addition, it is expected that the gradual increasing PMMA seeding induces the gradual decreasing cavity $Q$ value as a result that the seeding particles obstruct propagation of a beam in the droplet, thereby reducing an effective optical pathlength. Because the cavity $Q$ value is proportional to mirror reflectivity and optical pathlength if the other parameters are kept constant, lasing characteristics at higher frequency (at shorter wavelength) with decrease of mirror reflectivity predicted and observed [see Fig. 8 in Ref. 18, in which the increase of predicted lasing frequency (blueshifted lasing wavelength) is linearly proportional to the decrease of mirror reflectivity (lower- $Q$ values), in RH-6G dyes] is similar to those at shorter wavelengths with increase of the seeding particles in the present results. Thus we have given a simple explanation for the blueshifted lasing spectra as being due to the monotonical decreases of the cavity $Q$ value (corresponding to the monotonical increases of the scattering particles in the droplet cavity).

Furthermore, according to the results discussed above, it is considered that the result shown in Fig. 3, which exhibits a tendency of a monotonical relationship between lasingwavelength decrease (blueshifted lasing) and increase of the round-trip time required to support lasing, is partly reasonable, because the loss in the spherical cavity could be assumed to be monotonically proportional to the increase of the quantity of the injected scatterers into droplets. Dye lasing in the droplets with lower- $Q$ values requires longer round-trip time to overcome the larger losses and to obtain the larger gain to support lasing, under the other losses are kept constant.

We have reported the expected observation of the time delays of emission depending on lasing wavelengths from a dye-doped liquid droplet with a spherical cavity, which contains injected scatterers for wavelength-tuning due to cavity- $Q$ controlling.

The authors gratefully acknowledge the valuable suggestions of Professor H. Inaba, Tohoku Institute of Technology.

${ }^{1}$ C. G. B. Garrett, W. Kaiser, and W. L. Bond, Phys. Rev. 124, 1807 (1961).

${ }^{2}$ H.-M. Tzeng, K. F. Wall, M. B. Long, and R. K. Chang, Opt. Lett. 9, 499 (1984).

${ }^{3}$ H.-B. Lin, A. L. Huston, B. L. Justus, and A. J. Campillo, Opt. Lett. 11, 614 (1986)

${ }^{4}$ R. E. Benner, P. W. Barber, J. F. Owen, and R. K. Chang, Phys. Rev. Lett. 44, 475 (1980).

${ }^{5}$ M. K.-Gonokami, K. Takeda, H. Yasuda, and K. Ema, Jpn. J. Appl. Phys. 31, L99 (1992).

${ }^{6}$ A. Biswas, H. Latifi, R. L. Armstrong, and R. G. Pinnick, Opt. Lett. 14, 214 (1989).

${ }^{7}$ H. Latifi, A. Biswas, R. L. Armstrong, and R. G. Pinnick, Appl. Opt. 29, 5387 (1990).

${ }^{8}$ H.-B. Lin, J. D. Eversole, and A. J. Campillo, Opt. Lett. 17, 828 (1992).

${ }^{9}$ R. L. Armstrong, J.-G. Xie, T. E. Ruekgauer, and R. G. Pinnick, Opt. Lett. 17, 943 (1992).

${ }^{10}$ R. L. Armstrong, J.-G. Xie, T. E. Ruekgauer, J. Gu, and R. G. Pinnick, Opt. Lett. 18, 119 (1993).

${ }^{11}$ J.-G. Xie, T. E. Ruekgauer, R. L. Armstrong, and R. G. Pinnick, Opt. Lett. 18, 340 (1993).

${ }^{12}$ H. Taniguchi, H. Yamada, T. Fujiwara, S. Tanosaki, H. Ito, H. Morozumi, and M. Baba, Jpn. J. Appl. Phys. 32, L58 (1993).

${ }^{13}$ H. Taniguchi, H. Yamada, T. Fujiwara, S. Tanosaki, and M. Baba, Rev. Sci. Instrum. 64, 628 (1993).

${ }^{14} \mathrm{H}$ Taniguchi, T. Fujiwara, H. Yamada, S. Tanosaki, and M. Baba, Appl. Phys. Lett. 62, 2155 (1993).

${ }^{15}$ H. Taniguchi, S. Tanosaki, H. Yamada, T. Fujiwara, and M. Baba, J. Appl. Phys. 73, 7957 (1993).

${ }^{16}$ P. Chylek, A. Biswas, M. A. Jarzembski, and V. Slivastava, Appl. Phys. Lett. 52, 1642 (1988).

${ }^{17} \mathrm{H}$. Taniguchi and H. Tomisawa, Appl. Phys. Lett. 65, 3305 (1994).

${ }^{18}$ M. J. Weber and M. Bass, IEEE J. Quantum Electron. QE-5, 175 (1969). 